\title{
Ultra-low feedback fibre end termination geometry for high power fibre source applications
}

\author{
J. S. P. Chan, P. Wang, J. K. Sahu and W. A. Clarkson \\ Optoelectronics Research Centre, University of Southampton, Southampton, SO17 1BJ, UK
}

Optical fibre end termination techniques are very important in the development of fibre-based optical communication systems and high-power fibre sources in laser, amplifier and superfluorescent configurations. There are many situations where unwanted optical feedback from a fibre end-facet can be very detrimental to the performance of the fibre source. For example, in laser configurations in which an external cavity is employed to extend the functionality (e.g. for Q-switching, mode-locking or wavelength tuning), then feedback from the fibre end-facet adjacent to the external cavity will compete with the feedback provided by the external cavity itself and hence may impact adversely on the laser output characteristics.

The standard methods for reducing the effective feedback reflectivity of an end-facet include the use of an antireflection (AR) coating, or an angle-polished or angle-cleaved facet. Anti-reflection coatings can only reduce the endfacet reflectivity to $\sim 10^{-4}$ and hence are of limited use. Polishing the fibre end-facet at angle so that light reflected by Fresnel reflection does not couple to guided modes of the core is a very popular and quite effective method for reducing feedback. In theory, a very high level of suppression of optical feedback can be achieved if the angle, $\theta$ between the facet surface normal and the fibre's longitudinal axis is larger than [arcsin (NA)]/2, where NA is the numerical aperture of the core. However, in practice, the process of polishing also results in imperfections on the facet surface, and hence scattering, which limits the minimum reflectivity routinely achievable to $\sim 10^{-5}-10^{-6}$. Cleaving has traditionally been considered as a superior method for fibre end preparation owing to the flat surfaces with relatively low scattering that can routinely be produced. However, angle-cleaving is generally more problematic and it is difficult to produce high quality angle-cleaved facets with the large diameter fibres that are routinely used in claddingpumped fibre configurations.

Here, we report an alternative approach for producing high quality fibre end terminations with ultra-low feedback reflectivity [1]. We refer to this approach as the 'twisted-end termination' technique and it requires an offset-core fibre, as shown in figure 1. The fabrication procedure is very straightforward. Firstly, the outer-coating is removed from the end section of the fibre and then this section of fibre is heated. The fibre is then twisted so that the core has a helical path in this region. The fibre is then perpendicularly-cleaved in the twisted region to produce a high quality end-facet (i.e. with low scattering loss) perpendicular to the fibre's longitudinal axis. As a result of the helical trajectory, the core's path is at an angle with respect to the facet normal, and hence light fed back by Fresnel reflection suffers a further loss which depends on the angle. The core angle is determined by the core offset and number of turns per unit length, and hence can be selected to reduce the level of optical feedback from the end-facet to the required level without the penalty of a high 'bend loss' due to the helical trajectory. This approach has the advantage over angle polishing that it is very simple to implement and the facet quality is generally much better than can be achieved by polishing.

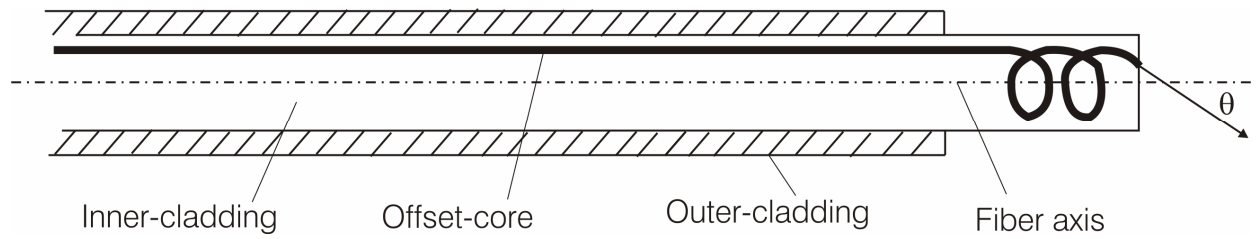

Fig.1: Twisted end termination in an offset core fibre

We have applied the twisted-end termination technique to a cladding-pumped Yb-doped offset-core fibre to suppress parasitic lasing for the efficient generation of high power amplified spontaneous emission (ASE) output. This required very low feedback reflectivity from the fibre end-facets. For a single-ended (single-stage) configuration, we obtained $63 \mathrm{~W}$ of ASE output. By comparison, a similar ASE source configuration with a standard angle-polished fibre end-facet produced only $27 \mathrm{~W}$ of ASE output before the threshold for parasitic lasing was reached. For a doubleended configuration with twisted end terminations at both ends of the Yb fibre, we obtained $110 \mathrm{~W}$ of ASE output, corresponding to a calculated single-pass gain of $\sim 70 \mathrm{~dB}$. The result indicates that the feedback reflectivity of the fibre end facet should be $\sim 10^{-7}$. Further experiments to confirm this are ongoing. The prospects for further reduction in facet reflectivity via this technique and the implications for high-power fibre devices will be discussed.

Reference:

[1] W. A. Clarkson, P. Wang, UK patent application “Optical fiber device”, application Number, 0600179.6, Jan. 2006 